\title{
Spectrum of MRI findings in traumaticknee
}

\author{
Mohi J. ${ }^{1}$, Bhatnagar S. ${ }^{2}$, Kaur N.K. ${ }^{3}$, Bansal N. ${ }^{4}$ \\ ${ }^{1}$ Dr. Jaswinder Kaur Mohi, Associate Professor, ${ }^{2}$ Dr. Simmi Bhatnagar, Assistant Professor, ${ }^{3}$ Dr. Navkiran Kaur, \\ Professor and Head, ${ }^{4}$ Dr. Neha Bansal, Junior Resident, all authors are affiliated with Department of Radio-Diagnosis, \\ G.M.C. Patiala, Punjab, India.
}

Corresponding Author: Dr. Simmi Bhatnagar, Assistant Professor, Dept. of Radio-Diagnosis, G.M.C. Patiala, Emailbhatnagarsimmi@yahoo.in

\begin{abstract}
Introduction: Injuries to the intraarticular structures like menisci and cruciate ligaments are diagnosed with high sensitivity and specificity by MRI as compared with arthroscopy, which is still regarded as the gold standard. The acutely injured knee is readily imaged for the detection of meniscal and ligamentous injuries. Aim: To describe the MRI features in various types of traumatic lesions andto analyze the types of knee joint abnormalities detected by MRI which will aid in making a proper diagnosis. Materials and Methods: A total of 51 patients with history of trauma to the knee joint, who were referred to radiology department from orthopaedic department of Rajindra hospital Patiala, suspecting internal derangement of knee, served as subjects for this study. The inclusion and exclusion criteriafor this study was fixed as well as MRI protocol which included T1 and T2 saggital, PD weighted sequences in axial, Coronal and Saggital, Fat Suppressed T2 or STIR sequences if needed. The different types of lesions of all components of knee joint were categorized separately and data was assessed. Results: Out of 51 patients who underwent MRI with history of trauma, 23 (45\%) patients had abnormal ACL and 28 (54.9\%) patients had normal ACL. PCL was found abnormal in only 3 (5.8\%) patients. MCL was abnormal in 5 (9.8\%), joint effusion was seen in maximum no of $44(86.2 \%)$ patients. Muscular pathology was seen in 1patient and bone pathology in $23(45 \%)$ patients. In medial meniscus tearsand lateral meniscus tears, posterior horn tears were the commonest. In ACL tears, acute partial tears were the commonest (61\%). Conclusion: In the setting of traumatic knee injury, MR imaging is the best noninvasive modality for the diagnosis of meniscal and ligamentous tears and to help guide the treatment of meniscal and ligament injuries,
\end{abstract}

Keywords: Menisci, Cruciate ligaments, Saggital and Parasaggital views, Complete and Partial tears.

\section{Introduction}

MRI allows superior soft tissue detail with multiplaner imaging capability that provides accurate evaluation of the intra and extra articular structures of the knee not demonstrated with any other imaging modalities currently available. The developments and advancements in MRI and the introduction of high resolution coils have provided a non-invasive, non-operator dependent, cost effective means to diagnose knee pathology.

MRI is well tolerated by patients, widely accepted by evaluating physicians and assists in distinguishing pathologic knee conditions that have similar clinical signs and symptoms [1,2]. The normal knee anatomy is displayed in 3 planes: sagittal, mid-coronal and axial.

Manuscript received: $18^{\text {th }}$ January 2018

Reviewed: $27^{\text {th }}$ January 2018

Author Corrected: $4^{\text {th }}$ February 2018

Accepted for Publication $9^{\text {th }}$ February 2018
Femoral, tibial and patellar cortices give a lowintensity signal and therefore looks dark. The soft tissue structures closely applied to bone namely the quadriceps tendon, the patellar tendon and the posterior cruciate ligament are also of low intensity. The bone marrow gives a high signal and hence looks bright. The midsaggital and parasaggital views provide the best visualization of the quadriceps and patellar tendons and of cruciate ligaments.

The menisci are best seen in parasaggital views as dark wedges. Behind the femoral condyle, the semimembranosus is seen as a black line which inserts onto tibia just beneath the posterior joint line [3]. Meniscal tears are seen as an area of linear, abnormally increased signal intensity within the meniscus, which extend to and communicate with an articular surface, best seen on short echo time (TE) sequences, such as SE 


\section{Original Research Article}

$\mathrm{T} 1$ or proton density weighted images or GE sequences [4], most common site being the posterior horn of the medial meniscus [5]. There are different types of meniscal tears like radial, horizontal and flap tears [6].It is very important to recognize the normal bowtie appearance of the body segments in both the medial and lateral meniscus on saggital images. Many abnormalities can be recognized easily by noting the absence of bowtie sign. Exceptions to this include a very large patient or very thin slices (the two-bowtie rule applies to slice thickness of $4-5 \mathrm{~mm}$ ) and discoid meniscus [7]. Ligamentous injuries appear as increased signal intensity on $\mathrm{T} 2$ and $\mathrm{T} 2$ star images in the acute tears or strains because of fluid or edema, others as discontinuity of the low signal intensity with or without loss of normal taut parallel margin, especially in thecomplete tears. Acute trauma requires $\mathrm{T} 2$ or fast spin-echo T2- weighted images [8].

There are certain primary features of complete ACL tear like dis-continuity with increased signal intensity at the site of tear, flat distal segment with high signal intensity near the femoral attachment, complete absence of the ligament with effusion and wavy ligament. Secondary signs of ACL tear include effusion, angulation of PCL, bone bruise, uncovered lateral meniscus, deep femoral notch, medial collateral ligament tear and meniscal tear. Similarly, complete and partial tears of PCL are there. Complete PCL tear is visualized as failure to identify PCL, inability to define its fibres with amorphous area of high signal intensity on $\mathrm{T} 1$ and $\mathrm{T} 2$ weighted MR images, or depiction of its fibres with a focal discrete disruption of all visible fibres. Partial PCL tears refers to PCLs that do not meet these criteria but demonstrated abnormal signal intensity within their substance or that have some intact and some discontinuous fibres.

Medial Colllateral Ligament tears are assigned grades corresponding to their MR appearance. Grade1 or sprain shows increased signal intensity in the soft tissues medial to MCL, Grade 2 or severe sprain or partial tear shows high signal intensity in the soft tissues medial to MCL but also high signal or partial disruption of the MCL and Grade 3 or complete tear showing disruption of the MCL.

\section{Materials and Methods}

Type and place of study- It was a prospective study which included 51 cases with history of trauma to theknee joint who were referred to radiology department of Rajindera hospital Patiala, suspecting internal derangement of knee. This dissertation evaluates various causes of derangement in traumatic knee joint. Study period was from Dec 2015 to Nov 2016 and it was a descriptive study.

Inclusion Criteria was all cases of suspected knee derangement with history of trauma.

Exclusion Criteria included patients referred from other hospitals for the sole purpose of getting an MRI scan done due to its non availability there and postoperative cases. The findings of meniscal tears were classified into area of involvement (anterior horn or posterior horn) as by Stoller et al [24] and grades of tears [24,25]:

Grade 1 meniscal tear is globular and does not communicate with the articular surface.

Grade 2 meniscal tear is linear in nature and remains within the substance of the meniscus not communicating with the articular surface.

Grade 3 meniscal tear has increased signal intensity within the meniscus that extends to the articular surface.

Grade 3a is a linear intrameniscal signal that abuts the articular margin.

Grade $3 b$ is a more irregular area of signal intensity that abuts the articular margin.

Grade 4 tears are menisci which are distorted in addition to the changes in grade 3

Types of tears were assessed as vertical, radial or parrot beak and bucket handle tears.

Ligaments were assessed in the following manner:ACL was considered to be intact [10] if it was visualized as a continuous linear band with low signal intensity that demonstrated normal orientation on proton density or T2- weighted images. ACLs with slight undulations, focal or diffuse thickening or amorphous appearance were also considered intact. The complete acute tear was suggested according to primary features as follows [9]:1. Discontinuity with increased signal intensity between segments or at the femoral or tibial attachments. 2. Flat or horizontal distal segment with high signal intensity near the femoral attachment. 3 . Complete absence of the ligament with effusion and high signal intensity in the mid-joint space.4.Wavy ligament.

Acute incomplete tears of ACL were suggested if it showed increased signal intensity (T2W1) with thickening and a normal course [10]. PCL tears were evaluated and graded as below [12]: 


\section{Original Research Article}

Complete tear of PCL was considered when there was failure to identify the PCL, inability to define ligamentous fibres with amorphous areas of high signal intensity on $\mathrm{T} 1$ and $\mathrm{T} 2$ weighted MR images in the region of PCL, or depiction of PCL fibres with focal discrete disruption of all visible fibres. Partial tear refers to PCLs that do not meet these criteria but demonstrated abnormal signal intensity within their substance or that have some intact and some discontinuous fibres. MCL and LCL tears were graded as Kaplan et al (07).

\section{Results}

Out of 51 patients who underwent MRI with history of trauma, 23(45\%) patients had abnormal ACL and 28 (54.9\%) patients had normal ACL. PCL was found abnormal in only $3(5.8 \%)$ patients. MCL was abnormal in 5 patients $(9.8 \%)$ and joint effusion was seen in maximum number of $44(86.2 \%)$ patients. Muscular pathology was seen in 1 patient and bone pathology related to trauma was seen in $23(45 \%)$ patients. While studying distribution of meniscal tears among medial and lateral meniscus, 15 patients $(44.1 \%)$ had medial meniscal tear and $06(17.6 \%)$ patients had lateral meniscus tear. While $13(38.2 \%)$ patients had abnormal both the medial and lateral meniscus. Of the 58 meniscal tears detected on MRI of the knee, $39(67.2 \%))$ tears involved the posterior horn, $15(25.8 \%)$ involved the anterior horn while $4(6.8 \%)$ involved the body of meniscus. Grade 2 tears of MM were seen in $23(69.7 \%)$ patients and grade 3 MM tears were seen in $10(30.3 \%)$ patients.

No patient was having grade 1 tear of MM. While studying distribution of tears among different parts of MM, tears involving posterior horn were seen in $24(72.7 \%)$ patients, tears involving anterior horn in $6(18.1 \%)$ and tears involving body of MM were seen in 3(9.09\%) patients. Of 25 lateral meniscal tears, 17(68\%) were grade 2 tears, $6(24 \%)$ were grade 3 tears and 2(8\%) were grade1 tear. Out of 25 lateral meniscal tears, 10 tears involved the anterior horn, 14 involved the posterior horn and 1 involved the body. Out of 23 patients having ACL tear, 14 patients (61\%) had acute partial tear, 4 patients $(17 \%)$ had acutecomplete tear, 5 patients $(22 \%)$ had chronic tears of ACL. In our study of 51 patients, 3 patients had PCL pathology in the form of complete or partial tear. Out of 3 patients having PCL tear, 2(67\%) of them had partial tear and $1(33 \%)$ had complete PCL tear. In our study, 5 patients were found to have abnormal MCL. Out of these 5 patients, 2 patients had grade 1 tear, 3 patients had grade 2 tear and none of the patients had grade 3 tear. Only 3 patients were found to have LCL tears, all 3 patients had grade 2 tear (partial tear). A total of 39 bone contusions were reported. Out of 39 bone contusions, $19(49 \%)$ were seen in tibia, $12(31 \%)$ in femur, 5(13\%) in patella and 3(8\%) in fibula. Bone contusions were more common in tibia than femur. Contusions in lateral femoral condyle [9] being more common than in the medial femoral condyle [3]. Out of 21 patients showing bone contusion, 3 patients also had MCL tears. Atotal of 7 bone fractures were seen in 6 patients out of which 5 (71.4\%) fractures involved the tibia,1 (14.3\%) fracture involved the femur, $1(14.3 \%)$ fracture involved the fibula.

Table-1: Distribution of patients according to kneepathology

\begin{tabular}{|c|c|c|}
\hline Pathology & No. of patients & Percentage \\
\hline ACL & 23 & 45 \\
\hline PCL & 03 & 5.8 \\
\hline Meniscus & 34 & 66.6 \\
\hline Collateral ligaments & 08 & 15.6 \\
\hline Bone & 23 & 45.09 \\
\hline Muscular & 01 & 1.96 \\
\hline Joint effusion & 44 & 86.2 \\
\hline
\end{tabular}

Joint effusion was seen in maximum percentage of patients followed by meniscal tears and other lesionsas given in table1

Table-2: Distribution of meniscal tears among medial and lateral meniscus.

\begin{tabular}{|c|c|c|}
\hline Meniscal Tears & Number of Patients & Percentage \\
\hline Medial Meniscus & 15 & 44.1 \\
\hline Lateral Meniscus & 6 & 17.6 \\
\hline Both Menisci & 13 & 38.2 \\
\hline Total & $\mathbf{3 4}$ & $\mathbf{1 0 0}$ \\
\hline
\end{tabular}

In meniscal tears, medial meniscal tears were seen most frequently. 
Original Research Article

Table-3: Distribution of tears in the anterior horn, posterior horn and body

\begin{tabular}{|c|c|c|}
\hline Meniscal Tears & Number of Tears & Percentage \\
\hline Anterior Horn & 15 & 25.8 \\
\hline Posterior Horn & 39 & 67.2 \\
\hline Body & 04 & 6.8 \\
\hline Total & $\mathbf{5 8}$ & $\mathbf{1 0 0}$ \\
\hline
\end{tabular}

Posterior horn was torn most frequently when comparison was made among different parts of menisci.

Table-4: Distribution of tears among parts of lateral meniscus.

\begin{tabular}{|c|c|c|}
\hline Part of Lateral Meniscus & No. of Tears & Percentage \\
\hline Anterior Horn & 10 & 40 \\
\hline Posterior Horn & 14 & 56 \\
\hline Body & 01 & 04 \\
\hline Total & $\mathbf{2 5}$ & $\mathbf{1 0 0}$ \\
\hline
\end{tabular}

Tears of posterior horn were most frequent and tears of body were the least common among parts of lateral meniscus.

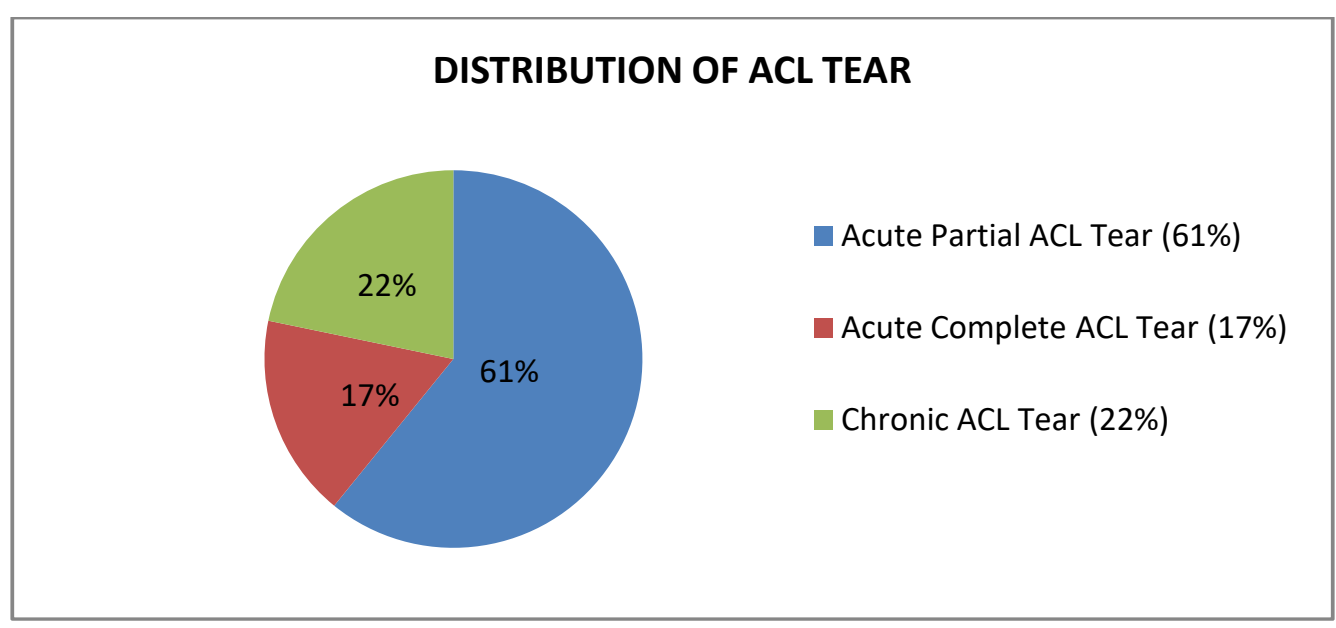

Figure-1: Pie chart showing different types of ACL Tears

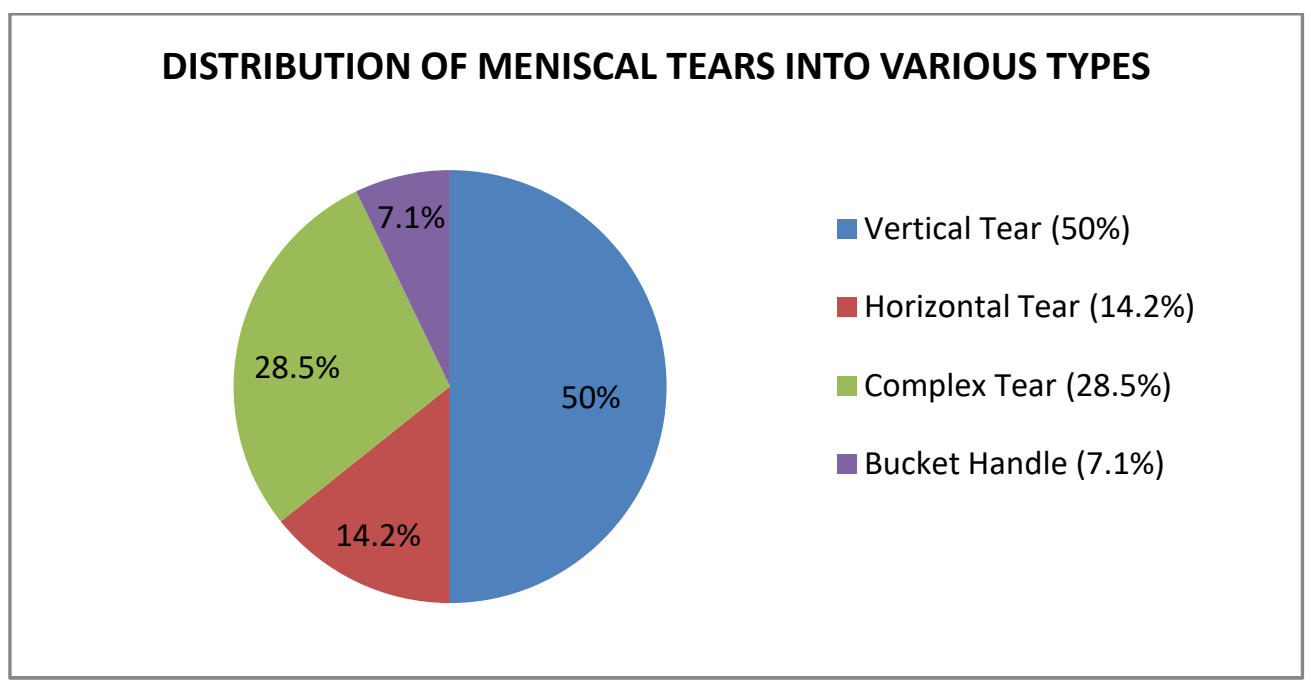

Figure-2: Pie chart showing distribution of different meniscal tears

Vertical tears were the commonest among different types of tears among menisci 
Original Research Article

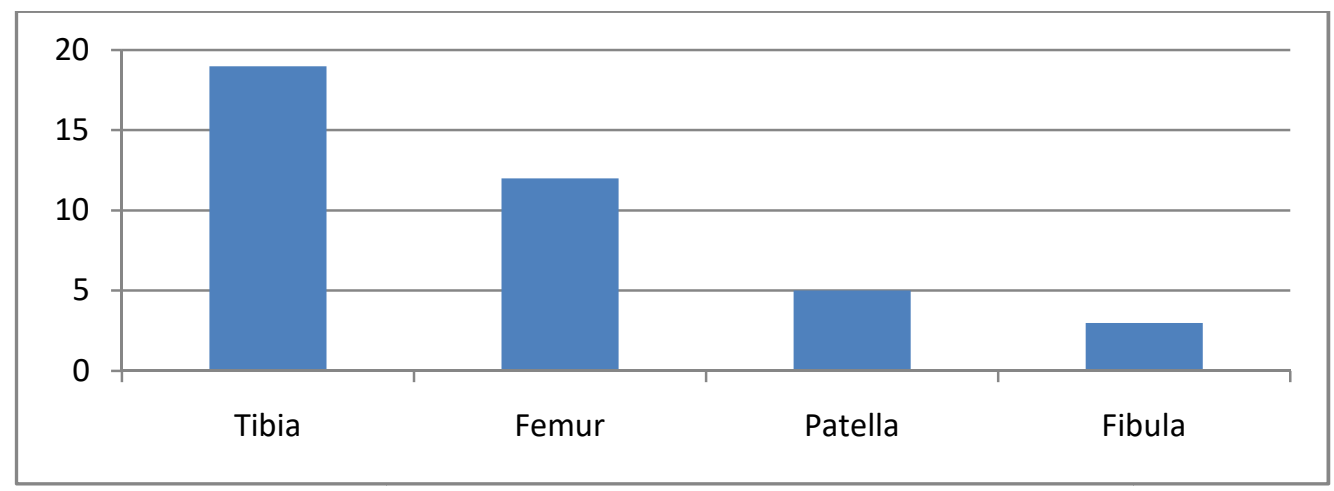

Figure-3: Bar chart showing distribution of bone contusions in different bones forming knee joint

Contusions in tibia were most frequent and in fibula least frequent while studying contusions among different bones forming knee joint.

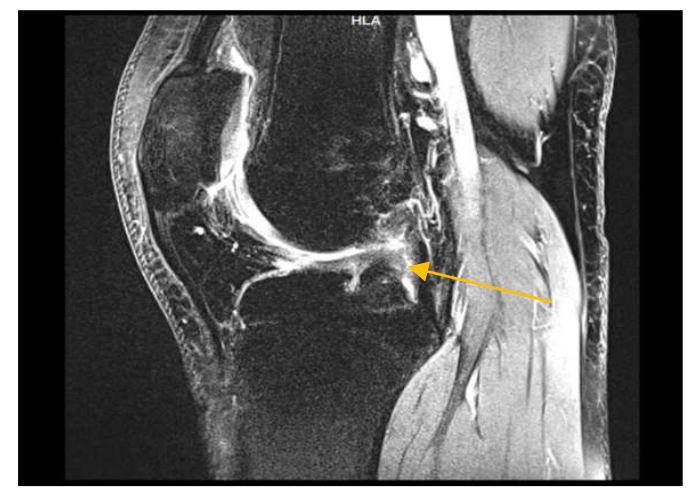

Figure-4: PD FS SAG Image showing complete ACL tear

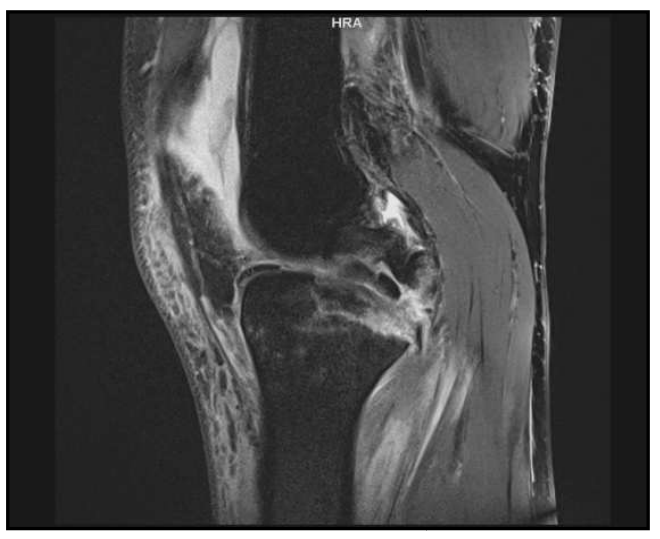

Figure-5: PD FS SAG image showing complete PCL Tear

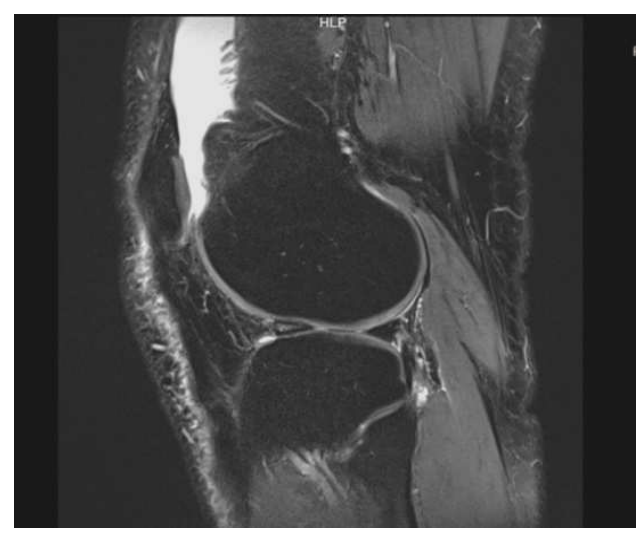

Figure-6: PD FS SAG Image Showing Grade-II Tear of Anterior Horn of Lateral Meniscus

\section{Discussion}

The present study included MRI of the knee joint of which 25 were left knee and 26 were right knee. In the present study of 51 patients, MRI of the knee was normal in only 2 patients (3.9\%). Hetta et al have observed similar results with 5 patients having normal MRI [20]. In the present study, injury to the menisciwas the commonest soft tissue abnormality encountered. This is in accordance with the study done by Adil Ismail Nasir [19] on 50 patients to investigate the accuracy of MRI of the knee for the detection of injuries of meniscus, cruciate ligaments and articular cartilage.In the present study of the 58 meniscal tears, 15 (25.8\%) involved the anterior horn, 39 (62.4\%) involved the posterior horn and only $4(6.8 \%)$ involved the body of meniscus. Crues et al [25] in their study also found meniscal tears involving the posterior horns more common (57\%) as compared to that of anterior horn $(16 \%)$. Similar results were also seen in study done by Singh et al [17] and Pame et al [23]. In the present study, of the 19 meniscal tears involving the lateral meniscus, 


\section{Original Research Article}

$1(5.2 \%)$ was classified as grade $1,12(63 \%)$ were of grade 2 and $6(31.5 \%)$ were of grade 3 . Of the 33 meniscal tears involving the medial meniscus, grade2 tears were the commonest. In the present study, 23 patients had ACL tears. Out of 23 patients having ACL tear, 14 patients had acute partial tear, 4 patients had acute complete tear and 5 patients had chronic tears of ACL. The results are comparable to the study done by Kamran et al [22]. An ACL tear was considered acute if the MRI examination was performed within 6 weeks of injury and chronic if MR examination was performed more than 6 weeks after injury as by Vahey et al [10]. In the present study, 3 patients had PCL pathologies. 3 patients having PCL tear and 1 patient having degenerative changes. Out of 3 patients having PCL tear, 2 of them had partial tear and 1 had complete PCL tear. It is comparable to the study done by Singh et al [11] in which 5.78\% patients showed PCL tears.

Similar results were also seen in study done by Hettaet al [20] and Pame et al [23] (2017). In the present study, bone contusion was found in 39 out of 51 patients (76.4\%). This result was not in agreement with the findings of Kamran et al [22] who reported bone contusions inanincidence of $28.3 \%$. In the present study, bone contusions were more common in tibiathan femur. Lateral femoral condyle (9/12) being more commonly involved than the medial femoral condyle (3/12) which is comparable to results of study done by Mathis et al [16] who reported that lateral compartment accounted for 65 of the 72 femoral condyle lesions and 64 of the 87 bone bruises of the tibial plateau. Bone bruises were most commonly associated with ACL and MCL injuries. In the present study, acute ACL tears were usually associated with bone bruise.

Mc Cauley et al [15], Arumugam et al [21] and Spindler et al [14] also reported a high incidence of bone bruise with ACL tear. In the present study, in 3 patients bone bruises were associated with MCL tear. Itis in concordance with the study done by Yoon et al [18] who found that,out of 86 patients with arthroscopically proven ACL tears, prevalence of bone contusions was $68 \%, 24 \%$ and $26 \%$ in the lateral femoral condyle, lateral aspect of tibia, medial femoral condyle and medial aspect of tibial plateau respectively. Prevalence of MCL injuries was $22 \%$ in their study.

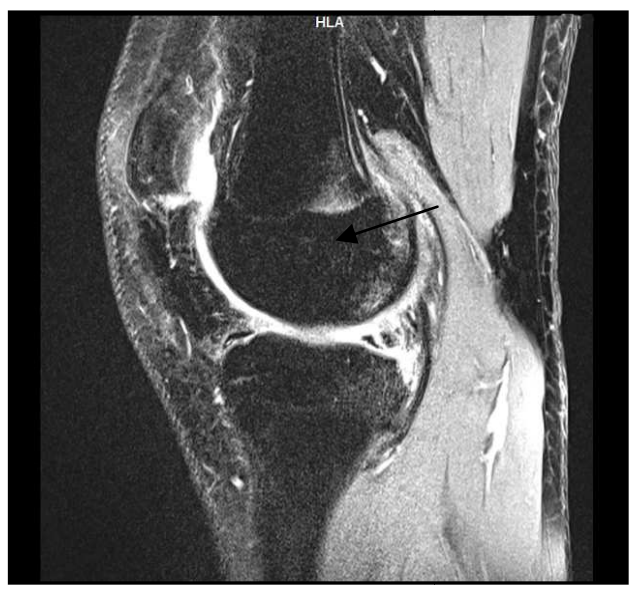

Figure-7: PD FS SAG Image showing complex tear of posterior horn of medial meniscus

\section{Conclusion}

From the present study we conclude that Magnetic Resonance Imaging is an accurate, noninvasive technique for examination of soft tissues and osseous structures of the knee.Studies have shown that it is cost effective when used to evaluate patients with history of knee trauma. In the setting of traumatic knee injuries, MR imaging is the best noninvasive modality for the diagnosis of meniscal and ligament tears. Familiarity with the normal anatomy and common pitfalls reduces errant interpretations but does not eliminate them completely. To help guide the treatment of meniscal and ligament injuries, the MR report should thoroughly

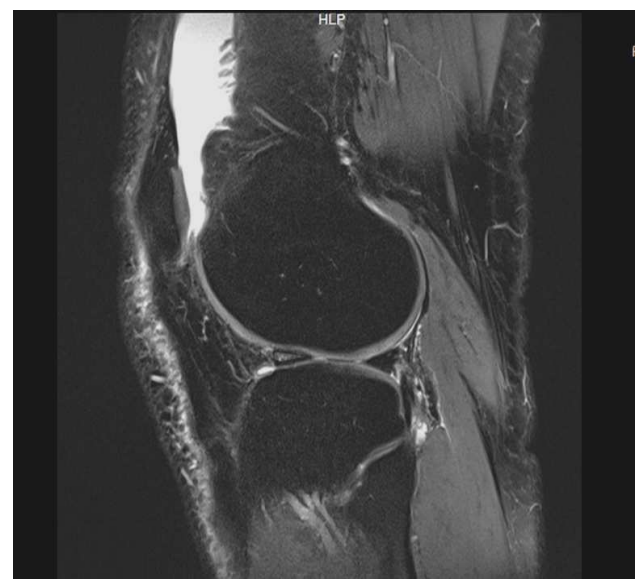

Figure-8: PD FS SAG image showing grade 2 tear of anterior horn of lateral meniscus

describe tears and not simply indicate whether a meniscal tear is present. The present study also demonstrates a valuable role of MR imaging in the examination of a wide spectrum of chronic knee abnormalities associated with acute trauma.

MR imaging depicts the anatomy of the knee joint without need for intravenous contrast agents or joint manipulation. Its accuracy in the diagnosis of meniscal tears is high as well as in the evaluation of ACL.

Funding: Nil, Conflict of interest: None. Permission of IRB: Yes 


\section{References}

1. Prickett WD, Ward SI, Matava MJ. Magnetic resonance imaging of the knee. Sports Med. 2001; 31 (14): 997-1019.

2. Kean DM, Worthington BS, Preston BJ, Roebuck EJ, McKim-Thomas H, Hawkes RC, Holland GN, Moore WS. Nuclear magnetic resonance imaging of the knee: examples of normal anatomy and pathology. $\mathrm{Br} \mathrm{J}$ Radiol. 1983 Jun; 56 (666): 355-64.

3. Soudry M, Lanir A, Angel D, Roffman M, Kaplan N, Mendes DG. Anatomy of the normal knee as seen by magnetic resonance imaging. Bone and Joint Journal. 1986; 68 (1):117-20.

4. Escobedo EM, Hunter JC, Zink-Brody GC, Wilson AJ, Harrison SD, Fisher DJ. Usefulness of turbospinecho MR imaging in the evaluation of meniscialtears: comparison with a conventional spin-echosequence. AJR Am J Roentgenol. 1996 Nov; 167 (5):1223-7.

5. Nguyen JC, De Smet AA, Graf BK, Rosas HG. MRimaging-baseddiagnosis and classification of meniscaltears. Radiographics. 2014 Jul-Aug;34(4): 98199. doi: 10.1148/rg.344125202.

6. Dandy DJ. The arthroscopic anatomy of symptomatic meniscal lesions. Bone and Joint Journal. 1990;72 (4): 628-33.

7. Kaplan AP, Helms CA, Dussault R. Anderson MV, Major NM: Musculoskeletal MRI. WB Saunders Company, Philadelphia 2009; 323-45.

8. Mansour MAM, Ahmed RM, Alaaibrahim, Elhussein $\mathrm{N}$, Aljuaid SA. Magnetic resonance imaging diagnostic procedures for knee joint injuries. IOSR-JNHS. 2015;4 (2): $37-46$.

9. Brandser EA, Riley MA, Berbaum KS, EL-Khoury GY, Bennett DL. MR imaging of anterior cruciate ligament injury: independent value of primary and secondary signs. AJR. American Journal of Roentgenology. 1996 Jul;167(1):121-6.

10. Vahey TN, Broome DR, Kayes KJ, Shelbourne KD. Acute and chronictears of the anterior cruciate ligament: differential features at MR imaging. Radiology. 1991; 181(1): 251-3.

11. Sonin AH, Fitzgerald SW, Friedman H, Hoff FL, Hendrix RW, Rogers LF. Posterior cruciate ligament injury: MR imaging diagnosis and patterns of injury. Radiology. 1994;190 (2):455-8.

12. Muller W. The Knee: Form, Function, and Ligament Reconstruction ( $1^{\text {st }}$ edn.). Springer-Verlag 1983; 187-90.

13. Recht MP, Applegate G, Kaplan P, Dussault R, Schweitzer M, Dalinka MK, Resnick D. The MR appearance of cruciateganglion cysts: a report of 16 cases. Skeletal Radiology. 1994 Nov; 23(8):597-600.

14. Spindler KP, Schils JP, Bergfeld JA, Andrish JT, Weiker GG, Anderson TE, Piraino DW, Richmond BJ, Medendorp SV. Prospective study of osseous, articular, and meniscallesions in recent-anterior cruciate ligament tears by magnetic resonance imaging and arthro-scopy. The American Jourrnal of Sports Med. 1993; 21(4): 551-7.

15. McCauley TR, Moses M, Kier R, Lynch JK, Barton JW, Jokl P. MR diagnosis of tears of anterior cruciate ligament of the knee: importance of ancillary findings. AJR American Journal of Roentgenology. 1994; 162 (1): 115-9.

16. Mathis CE, Noonan K, Kayes K. "Bone bruises" of the knee: a review. Iowa Orthopaedic Journal. 1998; 18:112-7.

17. Singh JP, Garg L, Shrimali R, Setia V, Gupta V. MR Imaging of knee with arthroscopic correlation in twisting injuries. Indian journal of radiology and imaging. 2004;14 (1):33-40.

18. Yoon KH, Yoo JH, Kim KI. Bonecontusion and associatedmeniscal and medialcollateral ligament-injury in patients with anterior cruciate ligament rupture. J Bone Joint Surg Am. 2011;93 (16):1510-8.

19. Nasir AI. The role of Magnetic Resonance Imaging in the Knee Joint Injuries. International Research Journal of Medical Sciences 2013;1 (5):1-7.

20. Hetta W and Niazi G. MRI in assessment of sports related knee injuries. The Egyptian Society of Radiology and Nuclear Medicine 2014;45 (4):11531161.

21. Arumugam V, Ganesan GR, Natarajan P. MRI Evaluation of Acute Internal Derangement of Knee. Open Journal of Radiology 2015;5: 66-71. 


\section{Original Research Article}

22. Sohail K, Ayesha H, Shireen K, Zahir S, Ambreen S, Rehana B. Role of MRI in painful knee. Ann. Pak. Inst. Med Sci. 2015;11 (3):137-41.

23. Pame M, Gayan M, Hazarika K, Roy DKR. MRI evaluation of painful knee joint- the correlation of multiple coexisting pathologies, age and sex. J. Evid. Based Med. Healthc. 2017;4(18):1019-27.
24. Stoller DW, Martin C, Crues JV 3rd, Kaplan L, Mink JH. Meniscaltears: pathologic correlation with MR imaging. Radiology. 1987;163(3):731-5.

25. Crues JV, Richard R, Morgan FW. Meniscal pathology: The expanding role of magnetic resonance imaging. Clinical orthopaedics and related research $1990 ; 252: 80-86$.

\section{How to cite this article?}

Mohi J, Bhatnagar S, Kaur N.K, Bansal N. Spectrum of MRI findings in traumaticknee. Int J Med Res Rev 2018;6 (02):85-92. doi:10.17511/ijmrr. 2018.i02.04. 